\title{
Texton Features and Deep Belief Network for Leaf Disease Classification
}

\author{
M Anandkumar \\ Department of Computer Science \\ Adigrat University, Ethiopia \\ anandmkumar15@gmail.com
}

\begin{abstract}
A world without agriculture would be very difficult for all living things because agriculture is an important part of our daily life. Day by day, agriculture is destroyed due to water scarcity, flood, pollutions, soil erosion, deforestation, plant or leaf disease, and so on. The main impact of agriculture is leaf disease or plant disease, which is caused by some kinds of fungus, bacteria, and viruses. If the farmers do not take proper care, then it causes more problems and reduces the quantity and quality of the product. This is the main reason that the detection of leaf disease is very important. In this paper, the tomato leaf disease classification is performed by the Deep Belief Network (DBN). The DBN is mainly focused to classify leaf diseases and improves the quality and production quantity of tomatoes. This method filters the unwanted signal from an input image by Gaussian filter, and then, the Region of Interest (ROI) reduces the pixels of the image. After that, the feature extraction extracts the received data, and finally, the DBN classification system classifies the leaf disease. The implementation and analysis of the proposed method exposed that the DBN classifier for tomato leaf disease classification yields a better performance of $98.97 \%$.
\end{abstract}

Keywords: Deep Belief Network (DBN), Gaussian filter, Region of Interest (ROI), Feature extraction, Leaf disease classification.

\section{Introduction}

The backbone of the Indian economy is agriculture because it provides much economic growth to the country, and more than seventy percent of Indian people are live dependent upon agriculture [1]. To be honest, in this world, most of the countries are depending on agriculture because the world without agriculture would be very difficult to survival for all living things. The main purpose of agriculture is raising livestock, cultivating the soil, and growing crops, plants, trees, and species. It helps to produce the products from the animal and plant products (leather, cotton, wool, foods, silks, and so on) for people to use. Nowadays, pollution, natural disaster, industries, development of the area, and so on creates an impact on the development of agriculture [2]. The major problem of agriculture is plant disease or leaf disease. If the farmers not taking proper care of the leaf disease, then it will affect the quality and productivity of the products such as fruits, flowers, leaves, and so on [3]. Leaf diseases are caused by various bacteria, fungus, and viruses. Every year in global harvest, 10-16\% losses are caused due to plant diseases, valued at US $\$ 220$ per year [4].

In agriculture, many medicinal plants such as tulsi/basil, mint, coriander, lemongrass, aloe vera, and so on play a virtual role in the medical field. According to World Health Organization (WHO), 80\% of the people in the world yet rely on plant-based traditional medicine because natural products are lower cost, toxic-free, and have fewer side effects than allopathic medicine [5]. The quality of the leaf is very important in medicinal plants. The leaf disease is a threat to the production and economic status in the worldwide agriculture industry [6]. The plants are mostly affected by the leaf spots, spores, rusts, downy mildew, powdery mildew, leaf blights, and so on. In India, tomato is one of the adopted vegetables but the tomato vegetables are affected by more than 20 tomato leaf disease that causes economic loss. The cultivation period of tomato plants is mostly done in the summer and winter seasons. The tomato plants are affected by leaf disease caused by viruses, bacteria, pests, and fungi [10]. Generally, the professionals who know about the tomato leaf disease can identify the type of disease in the tomato plants but that method requires a large team of experts. In this method, the farmer should give a lot of money to the company experts, and it takes too much time to make the process [7]. In another word, it is useless for 
monitoring the large area of agricultural land continuously by the specialist. So the automatic leaf disease classification is necessary to detect the tomato leaf disease [8]. In this technology world, there were a lot of techniques are available to classify leaf disease. However, not all leaf disease classification methods gave accurate results.

The main intention of the research is to classify the leaf disease based on the captured image of leaves by using Deep Belief Network (DBN). Initially, the digital camera captures the image of the plants or tree leaves to classify the disease. Once the image of the leaf is captured, then the image is passed to the preprocessing system for removing the unwanted noise from an image by Gaussian filter. After that, the pixels are reduces by the ROI thresholding, and the feature extraction minimizes the complexity of leaf disuse classification. Finally, the classification system classifies the result by using DBN.

The major contribution of the paper:

- Proposed Deep Belief Network (DBN) for leaf disease classification: The leaf disease classification is achieved using the proposed DBN for which the features considered are based on the techniques, such as Local Binary Pattern (LBP) and texton.

The paper is organized as follows: section 2 elaborates the literature review of the existing leaf disease classification. Section 3 elaborates on the proposed method of leaf disease classification using DBN. Section 4 elaborates on the experimental results and discussion, and finally, section 5 elaborates on the conclusion.

\section{Literature review}

This section illustrates the literature review of leaf disease classification techniques. The negative sides of existing leaf disease detection techniques are considered to create a novel approach for classifying the disease of a plant leaf or tree leaf in the agricultural land.

Hee-Jin Yu et al. [9] developed a deep learning architecture for identifying the apple leaf disease. It includes the model of the leaf spot attention mechanism for identifying the leaf disease with more accuracy. In this method, the feature segmentation sub-network is divided into the input images into the spot area, background, and leaf area in the feature map, and it is connected to the spot-aware classification sub-network. It increased the discriminative power by using the mechanism of leaf spot attention. The result shows that this method identified the apple leaf disease but not accurate segmentation. Haridas D. Gadade et al. [10] developed a segmentation-based approach for classifying the tomato leaf disease and severity measurement of the disease. The technique of leaf disease detection comprises various stages such as pre-processing system, segmentation, extraction, training, and SVM classification. The color-based thresholding method on HSV image is used for the segmentation of the tomato leaf diseased area. This method identified the disease on a tomato leaf and measured the severity level of the disease in the leaf. Rizqi Amaliatus Sholihati et al. [11] introduced the deep learning method by using VGG16 and VGG19 Convolutional Neural Network (CNN) architecture for classifying and identifying the healthy and disease infected potato leaf. In this research, CNN comprises data acquisition, pre-processing, augmentation, and classification. The result shows that this method is achieved an average accuracy of $91 \%$. However, the noise is occurred when more than one leaf presented in the frame. Surampalli Ashok et al. [12] developed the identification of disease in the tomato plant leaf by using the image processing method. In this method, the captured image is classified as the healthy leaf or disease affected leaf. Then the information of the image is stored in the database for further analysis and classification. The leaf disease is classified by several connected layers in the steps of subsequent. The result shows that this research achieved 98\% accuracy. Shreya Ghosal et al. [13] introduced transfer learning for developing a deep learning model to classify the rice leaf disease. In this method, VGG-16 Convolutional Neural Network (CNN) architecture is trained so it classified the rice leaf disease. The result shows the $92.46 \%$ accuracy level but it does not improve the accuracy and not decreasing the loss of validation data. Stefania Barburiceanu et al. [14] introduced the convolution neural network for predicting the type of disease. CNN has followed deep learning techniques and image processing. In this research, the algorithm is executed by using Python language that utilizes Flask as the framework of the micro web for GUI. It printed the predicted leaf disease when the user gives the image as an input. The result shows more than $70 \%$ accuracy but the problem is some images are not clear and some images are damaged. 


\section{Proposed Method of Leaf Disease Classification Using the Deep Belief Network Based on the Captured Image}

The main objective of this paper is to classify the leaf disease using DBN. To enable effective detection, the captured image is filtered by the Gaussian filter and then processed to extract the feature extraction. Based on the features, such as LBP and texton, the disease of the leaf is classified by using DBN.

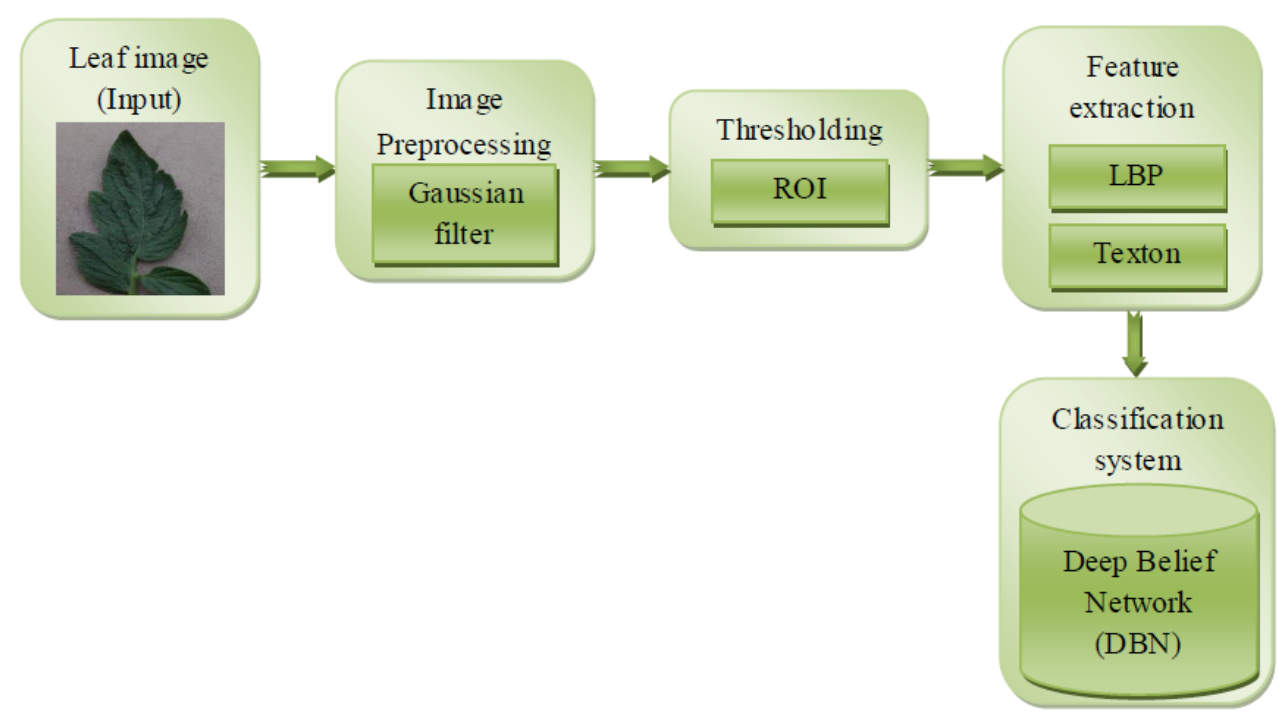

Fig.1 Block diagram of Leaf disease classification

This section explains the developed leaf disease classification to classify the leaf disease and healthy leaf based on the given input. Fig. 1 illustrates the block diagram of the proposed leaf disease classification approach. The proposed approaches include Gaussian filter, ROI thresholding, feature extraction, and classification. Initially, when the user captures the image of the leaf, then the image is considered as an input and passes to the preprocessing system. The pre-processing system is used for filtering unwanted noises from an input image by using the Gaussian filter. After that, the input image is passed to the ROI thresholding, and it reduces the pixel of the input image. Then the information of the input image is passed to the feature extraction. The feature extraction reduces the complexity of the image for further process, and it comprises LBP and texton. Afterward, the extracted input image is passed to the classification system. The classification system comprises a Deep Belief Network (DBN), and it classifies the input image as the diseased leaf or health leaf based on the extracted data.

\subsection{Image Preprocessing}

Initially, the tomato leaf image is subjected to image preprocessing, which is used to clean the data of the input image. The preprocessing method is compulsory before training deep learning models. The main objective of the preprocessing method is to minimize, or eliminate the noises present in the input image and improves the quality of the input image for further process.

\subsubsection{Gaussian Filter}

In the preprocessing method, the Gaussian filter is used for filtering the image of tomato leaf and removing unwanted noise and signals from the input image for improving the enhancement of the input image. It reduces the contrast of the image and blurs the edges of the images for better quality. The Gaussian filter makes the hidden characteristics of the tomato leaf image visible. After that, the input image is passed to the thresholding.

\subsection{Thresholding}

Thresholding is the simplest method of image segmentation. The segmentation process is a complicated task because of the diversity and difficulty of images. It converts an image into multiple regions or multiple segments. Thresholding is used to replace each pixel that presents in an input image through a black pixel or white pixel for making the image easier to analyze the data for classifying the disease. It uses the Region of Interest (ROI) to perform some process on an image. 


\subsubsection{Region of Interest (ROI)}

Region of Interest (ROI) is used to define the boundaries of leaf images for measuring the size of the disease. It removes the unwanted pixels and focuses on the particular area of an image to classify the disease. To identify an object in the input image the ROI has been applied. In this research, the ROI mask is applied to the input image. The parts of the input image that recognized by the ROI mask is set as " 1 " and the non-ROI mask area is set as "0". The ROI mask area is passed to the feature extraction process.

\subsection{Feature Extraction}

The most important process in leaf disease classification is feature extraction. Feature extraction is used in many image processing applications. It is the process of the reduction of dimensional in an input image. In this research, the feature extraction extracts the data by Local Binary Pattern (LBP) and texton. Texture, color, edges, morphology are the features of extraction that is used in tomato leaf disease classification.

\subsubsection{Local Binary Pattern (LBP)}

The method of Local Binary Pattern (LBP) [18] is a simple but very efficient texture operator that labels the interior image pixel by thresholding the region of every pixel and thinks the output as a binary number. Because of its computational simplicity and discriminative power, the operator of LBP texture is very useful in many applications.

\subsubsection{Texton}

The term "texton" was proposed by Julesz [14] in 1981. In texture analysis, texton plays an important role. Texton is defined as an emergent pattern or set of blobs by sharing the common property all over the input image. Texton and color diversification have a close relationship with image feature extraction. The texton difference may form different image features. The extracted data of LBP and texton is passes to the classification system for identifying the tomato leaf disease.

\subsection{Classification System}

The final step of this research is the classification system wherein an input image is classified based on the predicted data. In the previous method, there were a lot of classifications systems are executed such as Deep Neural Network (DNN), Convolutional Neural Network (CNN), Artificial Neural Network (ANN), Decision tree, Linear Regression Analysis (LRA), and so on. In this method, the processed image is classified as the healthy leaf or diseased leaf by using Deep Belief Network (DBN).

\subsubsection{Deep Belief Network (DBN)}

A Deep Belief Network (DBN) is considered as a stack of Restricted Boltzmann Machine (RBM), wherein each RBM layer exchanges the data through both the previous layer and subsequent layer. Any single layers' nodes are do not exchange the data through each other laterally. The RBMs stack ends with the Softmax layer for creating a classifier. In DBN, other than the first and final layers each layer has a double work: it acts as a hidden layer for the nodes that come before it; as a visible (input) layer for the nodes that come after. DBN is used to classify, cluster, recognition, and generate motion-capture data, video, and images, and on the other hands, DBN is applicable for the classification. Hence, DBN is used in this research for tomato leaf disease classification. 


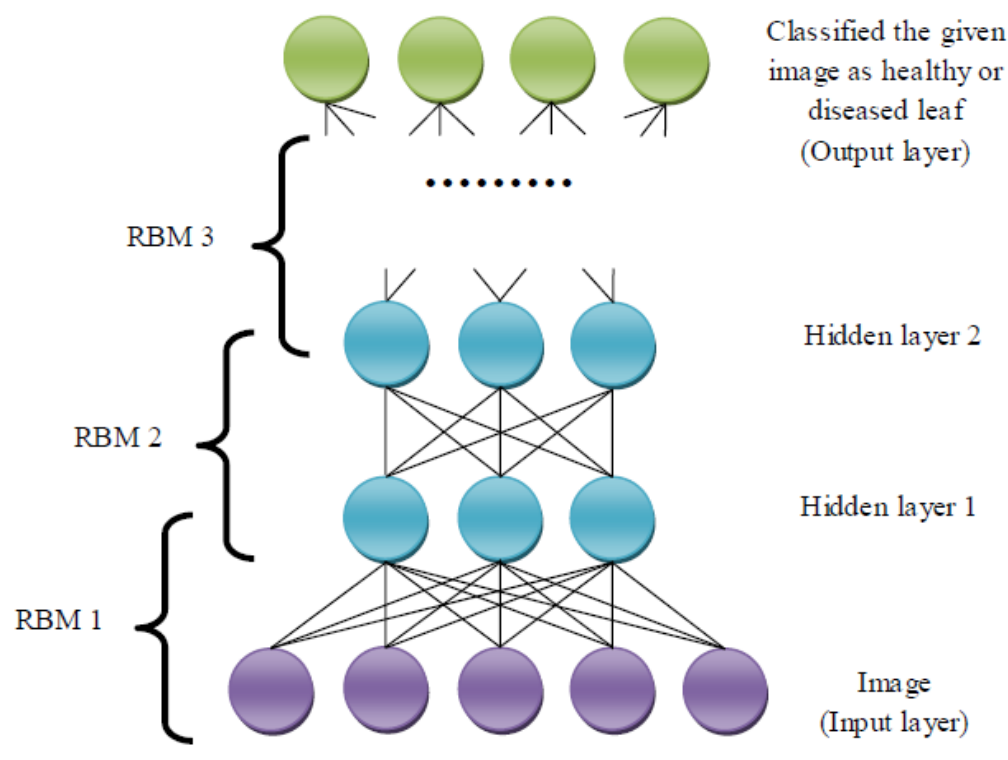

Fig.2. Architecture of Deep Belief Network (DBN)

The extracted data are fed to DBN for classifying the tomato leaf disease. The input image to the input layer is the features selected from the LBP and Texton that are processed further in the hidden layers to enhance the classification performance. Fig. 2 shows the architecture of the DBN. The classifier, DBN classifies the input image as a healthy or diseased leaf based on the information received from the extraction. It revealed the tomato leaf disease with more accuracy than other classifiers.

\section{Experimental Results and Discussion}

The experimental results and the discussion of a developed technique are described in this section. The experimental setup, dataset description, and comparative analysis are given below.

\subsection{Experimental Setup}

The execution of the developed leaf disease classification model is performed using MATLAB with Deep Belief Network (DBN) for classifying the tomato leaf disease.

\subsection{Dataset Description}

For each class, $75 \%$ of the samples were used for training, and $25 \%$ for testing. To estimate this research, we used the dataset with the images of diseased and healthy tomato leaves. The project of PlantVillage has collected a large database of many healthy and infected plant species, including tomato leaves. The PlantVillage dataset has images of healthy and diseased tomato leaves of 8 different types of leaf diseases [17]. We have used 8 different types of tomato leaves. Fig. 3 shows some samples from each category.

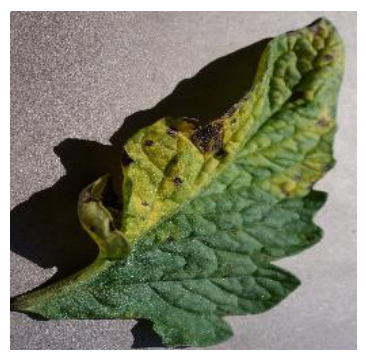

(a)

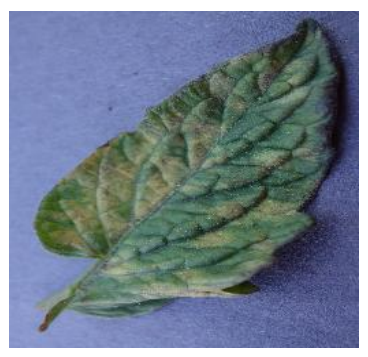

(b)

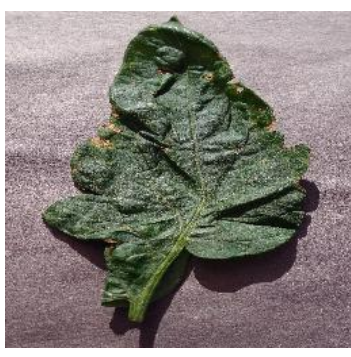

(c)

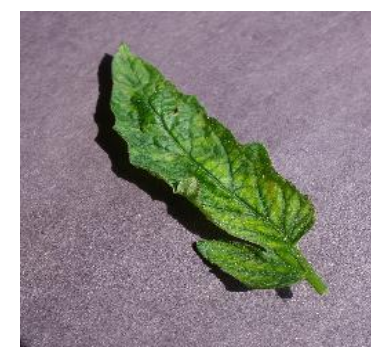

(d) 


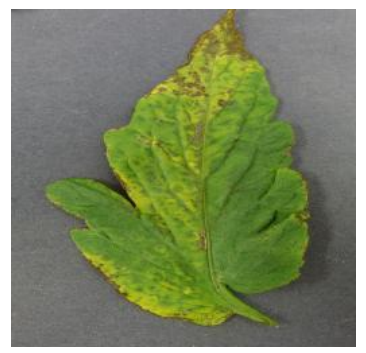

(e)

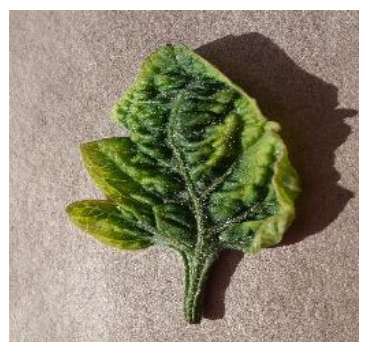

(f)

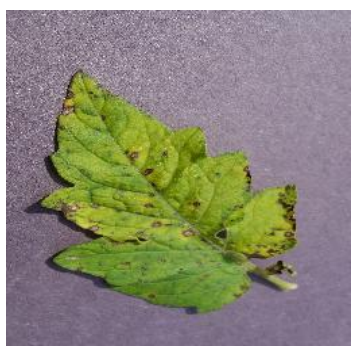

(g)

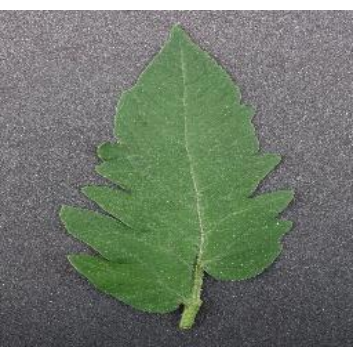

(h)

Fig.3 Samples of tomato leaves a) Blight, b) Leaf Mold, c) Target Spot, d) Mosaic Virus, e) Bacterial Spot, f) Yellow Leaf Virus, g) Septoria Leaf Spot, h) Healthy leaf

The PlantVillage database contains 16,012 images of the healthy and diseased tomato leaf [17]. The database classes and the total number of samples (6782) we were used for testing are given below:

$>$ Healthy tomato leaves (475 samples)

$>$ Tomato leaves affected by bacterial spot (1250 samples)

$>$ Tomato leaves affected by blight (953 samples)

$>$ Tomato leaves affected by leaf mold (242 samples)

$>$ Tomato leaves affected by the mosaic virus (869 samples)

$>$ Tomato leaves affected by septoria leaf spot (521 samples)

$>$ Tomato leaves affected by target spot (1334 samples)

$>$ Tomato leaves affected by yellow leaf curl virus (1138 samples)

Table.1. Accuracy of tomato leaf disease classification (Proposed method)

\begin{tabular}{ccc}
\hline S.No & Various types of Tomato leaves & Accuracy (\%) \\
\hline 1 & Blight & $98.88 \%$ \\
2 & Leaf mold & $98.65 \%$ \\
3 & Target spot & $98.98 \%$ \\
4 & Mosaic virus & $98.69 \%$ \\
5 & Bacterial spot & $99.61 \%$ \\
6 & Yellow leaf virus & $98.91 \%$ \\
7 & Septoria leaf spot & $98.45 \%$ \\
8 & Healthy leaves & $99.59 \%$ \\
\hline Total & & $98.97 \%$ \\
\hline
\end{tabular}

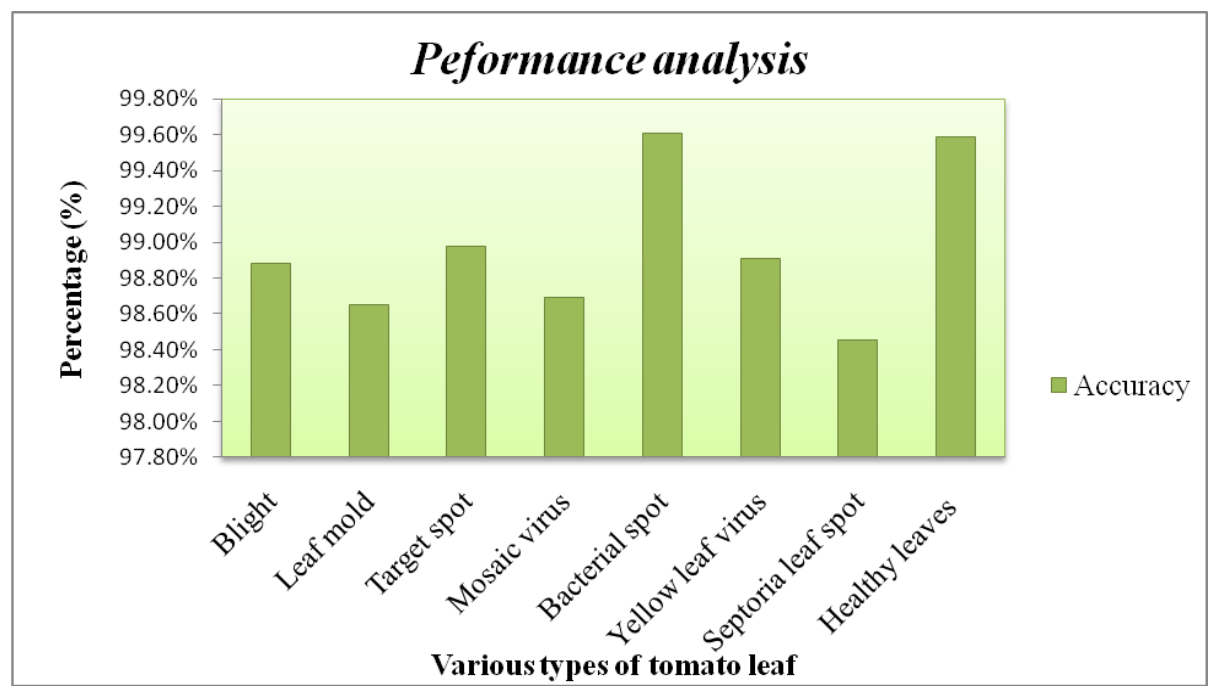

Fig.4. Performance analysis of the tomato leaf

Table.1. shows the accuracy of the proposed method. In this research, the proposed method shows clear development because of Deep Belief Network (DBN) and classified the tomato leaf as healthy or diseased with a very low error rate. The analysis proves that the tomato leaf disease classification produced a high accuracy rate of $98.97 \%$ is shown in Table 1 . Fig..4 shows the performance analysis of this research. Here, the accuracy of early \& late blight disease is $98.88 \%$, the accuracy of leaf mold disease is $98.65 \%$, the accuracy of target spot disease is $98.98 \%$, the accuracy of the mosaic virus is 
$98.69 \%$, the accuracy of the bacterial spot is $99.61 \%$, the accuracy of yellow curl leaf virus is $98.91 \%$, the accuracy of septoria leaf spot is 98.45 , and the accuracy of healthy leaves is $99.59 \%$.

\subsection{Comparative Analysis}

The performance of the proposed method is analyzed by considering the existing technique like Convolutional Neural Network (CNN) classification [12], Artificial Neural Network (ANN) [15], Deep Neural Network (DNN) [16], and Leaf Spot Attention Network (LSA-Net) [9]. The performance of the developed Deep Belief Network (DBN) produced high accuracy rate than the above mentioned methods due to LBP and Texton extraction. It is classified by several hidden layers and reduces the error rate over the training and testing.

Table.2. shows the performance analysis of the existing method and the proposed method. The proposed method shows that the DBN produced a higher accuracy rate than previous methods. Fig. 5 is shown the performance analysis of various methods with the proposed method. It is concluded that the developed DBN shows clear improvement and classifies the tomato leaf accurately. It has a minimum error rate.

Table.2. Comparison Between Existing Methods And The Proposed Method

\begin{tabular}{lll}
\hline S.No & Leaf disease detection methods & Accuracy \\
\hline $\mathbf{1}$ & Convolutional Neural Network (CNN) & $98.12 \%$ \\
$\mathbf{2}$ & Artificial Neural Network (ANN) & $92.94 \%$ \\
$\mathbf{3}$ & Leaf Spot Attention Network (LSA-Net) & $89.4 \%$ \\
$\mathbf{5}$ & Deep Neural Network (DNN) & $95.75 \%$ \\
\hline
\end{tabular}

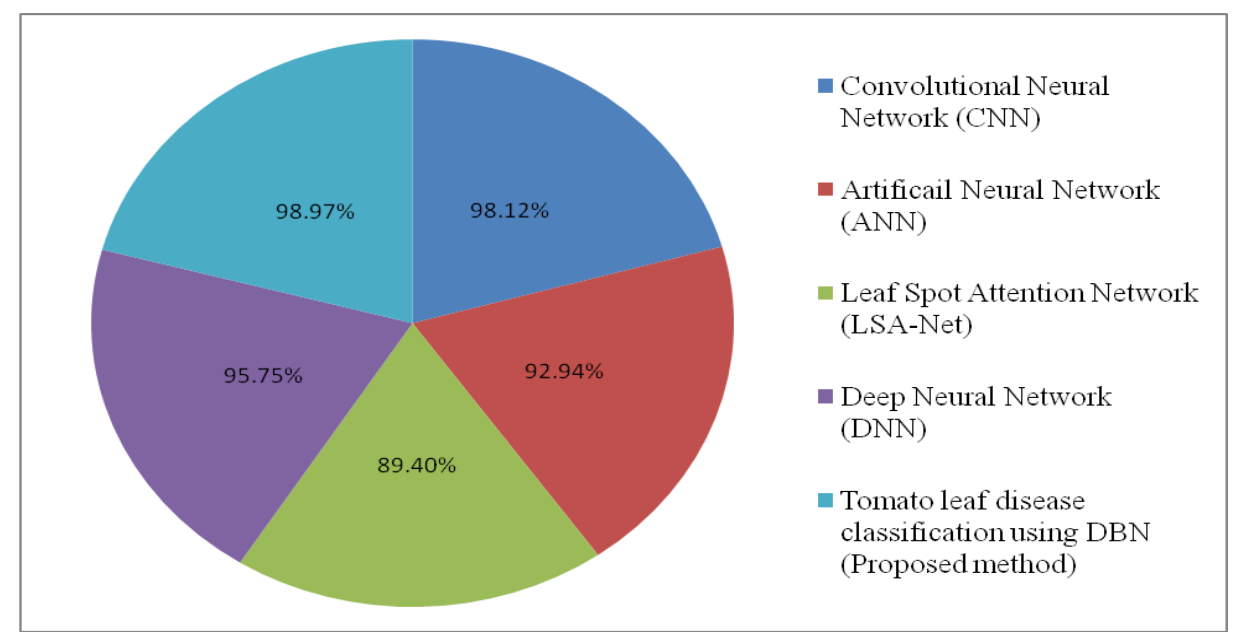

Fig.5. Performance analysis of various method with the proposed method

\section{Conclusion}

The future of farmers depends upon agriculture because it provides economic growth to the farmers. Unfortunately, due to leaf diseases, the quality and quantity of agricultural products are rapidly reduced. As a result, many farmers stop to cultivate agricultural products such as crops, tomatoes, rice, wheat, potato, and so on. Now the challenge is regarding the analysis of the leaf disease. Nowadays, the deep learning process plays a virtual role in leaf disease detection. Thus, Deep Belief Network (DBN) based tomato leaf disease classification is implemented and stands as a significant tool to the farmers for classifying the leaf disease. In this research, the disease of tomato leaf is classified based on the extracted data using DBN. The DBN classifier identified the leaf disease through the given data. It is revealed that the classification of tomato leaf disease is achieved $98.97 \%$ accuracy and in the future, more advanced methods will be executed for classifying the leaf disease. This research is not only for the classification of tomato leaf disease; it also executable in other crops and plants too for classifying the leaf disease. The proposed method also shows that the DBN yielded better performance than existing methods such as CNN, ANN, LSA-Net, and DNN model. 


\section{Compliance with Ethical Standards}

Conflicts of interest: Authors declared that they have no conflict of interest.

Human participants: The conducted research follows the ethical standards and the authors ensured that they have not conducted any studies with human participants or animals.

\section{References}

[1] Ashmi V K, Sahaya Edal Queen G, Anisha V, Sree Sankar J, Dr.D. Ramalingam, "Fire Detection and Irrigation Automation based on IoT", Conference Proceeding of International Conference on Innovative Research in Engineering, Science, Management and Humanities (ICIRESMH-2018), March 2018.

[2] Monu Bhagat, Dr. Dilip Kumar, Rehan Mahmood, Monu Kumar, Bibhudhendra Pati, "Bell Pepper Leaf Disease Classification Using CNN", IEEE, August 2020.

[3] Vijai Singh and A.K. Misra, "Detection of Plant Leaf Diseases using Image Segmentation and Soft Computing Techniques", Information Processing in Agriculture, October 2016.

[4] Siva K. Balasundram, Kamlesh Golhani, Redmond R. Shamshiri, Ganesan Vadamalai "Precision Agriculture Technologies for Management of Plant Diseases", Springer Nature, Plant Disease Management Strategies for sustainable Agriculture through Traditional and Modern Approaches, Sustainability in Plant and Crop Protection, Springer, Volume 13, pp: 259-278.

[5] Gittaly Dhingra, Vinay Kumar, Hem Dutt Joshi, "A novel computer vision-based neutrosophic approach for leaf disease identification and classification", Elsevier, Volume 135, PP: 782-794, March 2019.

[6] Karthikeyan N, Anjana M S, Anusha S, Divya R M, Asha Vinod, "Leaf Disease Detection using Image Processing”, IJISET - International Journal of Innovative Science, Engineering \& Technology, Vol. 7 Issue 5, pp: 60-66, May 2020.

[7] B. Rajesh, M. Vishnu Sai Vardhan, L. Sujihelen, "Leaf Disease Detection and Classification by Decision Tree", 2020 4th International Conference on Trends in Electronics and Informatics (ICOEI), IEEE, pp: 705-708, July 2020.

[8] Isaac Kofi Nti, Gyamfi Eric, Yeboah Samuel Jonas, "Detection of Plant Leaf Disease Employing Image Processing and Gaussian Smoothing Approach", International Journal of Computer Applications (0975 - 8887), Volume 162 - No 2, pp: 20-25, March 2017.

[9] Hee-Jin Yu and Chang-Hwan Son, "Leaf Spot Attention Network for Apple Leaf Disease Identification", IEEE/CVF Conference on Computer Vision and Pattern Recognition (CVPR) Workshops, pp: 52-53, 2020.

[10] Haridas D. Gadade and Dr. D. K.Kirange, “Tomato Leaf Disease Diagnosis and Severity Measurement”, IEEE, pp: 318-323, October 2020.

[11] Rizqi Amaliatus Sholihati, Indra Adji Sulistijono, Anhar Risnumawan, Eny Kusumawati, "Potato Leaf Disease Classification Using Deep Learning Approach”, 2020 International Electronics Symposium (IES), IEEE, pp: 392 397, November 2020.

[12] Surampalli Ashok, Gemini Kishore, Velpula Rajesh, S.Suchitra, S.G.Gino Sophia, B.Pavithra, "Tomato Leaf Disease Detection using Deep Learning Techniques”, 2020 5th International Conference on Communication and Electronics Systems (ICCES), IEEE, pp: 979-983, 48766, July 2020.

[13] Shreya Ghosal and Kamal Sarkar, "Rice Leaf Diseases Classification Using CNN With Transfer Learning", Proceedings of 2020 IEEE Calcutta Conference (CALCON), pp: 230-236, IEEE, June 2020.

[14] B. Julesz, "Textons, the elements of texture perception, and their interactions", Nature 290 (5802) 91-97, 12th March 1981.

[15] Robert G. de Luna, Elmer P. Dadios, Argel A. Bandala. "Automated Image Capturing System for Deep Learningbased Tomato Plant Leaf Disease Detection and Recognition", Proceedings of TENCON 2018, IEEE Region 10 Conference, 2018.

[16] Fuentes, S. Yoon, S. C. Kim, D. S. Park, “A Robust Deep-Learning-Based Detector for Real-Time Tomato Plant Diseases and Pests Recognition", Proceedings of TENCON 2018, IEEE Region 10 Conference (Jeju, Korea, 28-31, October 2018).

[17] PlantVillage Dataset, "https://www.kaggle.com/emmarex/plantdisease".

[18] Amer G. Binsaadoon and El-Sayed M. El-Alfy, "Gait-based Recognition for Human Identification using Fuzzy Local Binary Patterns", In: Proceedings of the 8th International Conference on Agents and Artificial Intelligence (ICAART 2016), Volume 2, pp: 314-321. 